\title{
Impacto de la gestión tecnológica sobre la capacidad de innovación en el sector restaurantero de Medellín
}

\author{
Alejandro Delgado-Cruz, Elva Esther Vargas-Martínez, \\ Juan Manuel Montes-Hincapié
}

Recibido: 23 de Diciembre de 2020

Aceptado: 16 de Febrero de 2021

https://doi.org/10.37610/dyo.v0i73.594

\section{Resumen}

El objetivo es analizar el impacto de la gestión tecnológica sobre la capacidad de innovación en el sector restaurantero de Medellín, Colombia. Se aplicó una encuesta a 139 directivos de empresas y se utilizó la modelación de ecuaciones estructurales por mínimos cuadrados parciales (PLS-SEM) para el tratamiento de datos. Los resultados señalan que en conjunto la estrategia tecnológica, las prácticas de $\mathrm{I}+\mathrm{D}$ y la asimilación tecnológica explican y predicen eficientemente la capacidad de innovación desde la estructura organizacional, el capital humano y las redes de colaboración. La gestión tecnológica aparece como una herramienta para potenciar las innovaciones de los restaurantes, favoreciendo su posicionamiento y competitividad en el mercado.

\section{Palabras clave}

Gestión tecnológica, capacidad de innovación, sector restaurantero

\section{Introducción}

En un entorno complejo y vulnerable por las transformaciones tecnológicas, las empresas tienen cada vez más dificultades para innovar y sobresalir en el mercado. Ante esto, la gestión tecnológica ofrece un nexo entre la estrategia competitiva y las oportunidades para el aprovechamiento de los recursos y las capacidades tecnológicas, al mismo tiempo de alcanzar un alto grado de innovación (Cetindamar, Phaal y Probert, 2016a; Huang, Wu, Lu y Lin, 2016). A pesar de ello y su progresiva importancia desde los años noventa, todavía es algo relativamente nuevo para las empresas de servicios, en especial, aquellas que por su naturaleza no son intensivas en tecnologías duras (Delgado, Vargas, Rodríguez y Montes, 2017a). Además, se ha reforzado la gestión tecnológica por concebirse como el área especializada en el estudio del fenómeno innovador, impulsado programas formativos para su desarrollo (Cetindamar, Phaal y Probert, 2016b; D’Mello, 2020; Mendoza, Barhate, Johnston y Jabarkhail, 2020).

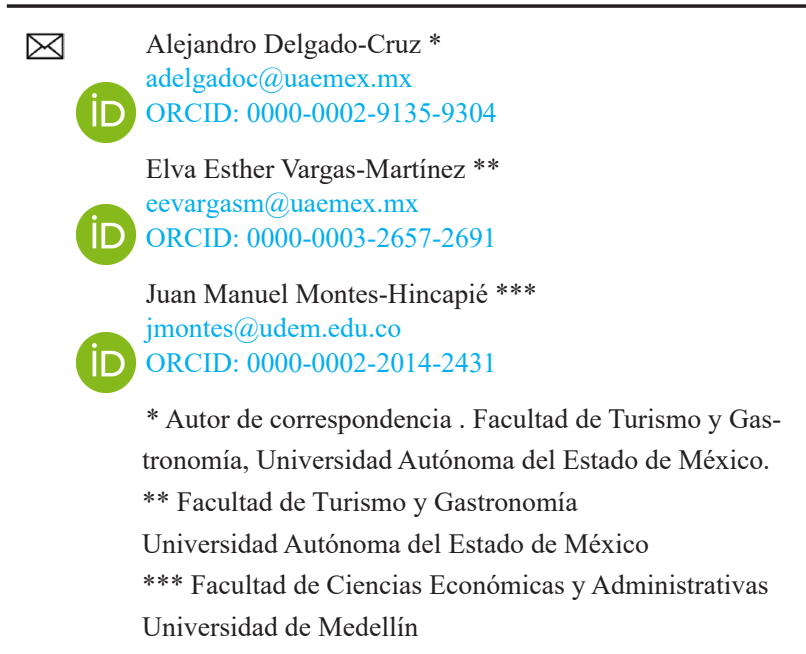

En particular, los restaurantes son empresas dedicadas al servicio de alimentos y bebidas. A pesar de ser en su mayoría micro, pequeñas y medianas empresas, para muchos países éstas representan un fuerte pilar económico y una gran fuente de empleo. Por ejemplo, en los Estados Unidos de América las ventas de los restaurantes suman un total de 863 mil millones de dólares al año, es decir, 4\% del Producto Interno Bruto (PIB); aparte de dar trabajo a 15.3 millones de personas (National Restaurant Association, 2019). En países europeos como España, el sector de la restauración y el alojamiento reportan ingresos de 123 mil millones de euros representando 6.2\% del PIB en el 2018, así como 1.7 millones de empleos (Hostelería de España, 2019). En el contexto de Latinoamérica, la industria restaurantera en México contribuye al 2\% del PIB y a la empleabilidad de 840 mil trabajadores (SECTUR, 2020). Mientras que en Colombia, los restaurantes dan trabajo a 99,227 personas (DANE, 2019) y junto con los hoteles contribuyen a $3.86 \%$ del PIB (CITUR, 2020).

Ante tal relevancia económica del sector restaurantero, no es de extrañar que el número de publicaciones científicas sobre su estudio va en aumento (DiPietro, 2017). En particular, los temas de investigación han versado sobre la capacidad de innovación y su relación con el emprendimiento, el rendimiento y los ingresos (Cho, Bonn y Han, 2019; Chou, Horng, Liu, Huang y Zhang, 2020; Delgado, Vargas, Rodríguez y Montes, 2018; Najib, Septiani y Nurlaela, 2020; Lee, Hallak y Sardeshmukh, 2016; Lee, Sardeshmukh y Hallak, 2016). Aunque también se ha prestado atención a temas emergentes como las plataformas de innovación abierta y la innovación sustentable (Salzberg, Gough y Suen, 2019; Yun, Park, Gaudio y Corte, 2020). 
Además, las evidencias empíricas señalan que la capacidad de innovación depende de la difusión y asimilación de las tecnologías en todas sus vertientes, que van desde aparatos y equipos para optimizar el desempeño productivo, hasta los conocimientos especializados que se adquieren para ofrecer bienes y servicios innovadores (Ahn y Seo, 2018; Kim, Choe y Hwang, 2020; Moreno y Tejada, 2019; Leung y Loo, 2020; Park, Lehto y Lehto, 2021; Seyitoğlu e Ivanov, 2020).

En el contexto colombiano, la ciudad de Medellín ha destacado por su esfuerzo y trabajo para propiciar la innovación en los diferentes sectores productivos. A tal grado de que en el año 2013, fue reconocida como "la ciudad más innovadora del mundo", por el Citigroup y el Wall Street Journal, gracias al fomento de políticas de inclusión social, el moderno sistema de transporte público y las acciones de cuidado ambiental (Villa y Melo, 2015). Bajo este esquema, Medellín asume su compromiso por continuar desarrollando mecanismos y plataformas para la innovación, mismas que se soportan de la ciencia y la tecnología.

Los restaurantes de Medellín han tenido un crecimiento y evolución similar que en otras regiones latinoamericanas. Las propuestas van desde conceptos vanguardistas como la cocina de autor y fusión, hasta aquellas que conservan la tradición de la gastronomía colombiana y de otras especialidades. Sin embargo, se enfrentan con el enorme reto de prevalecer en un mercado saturado de competidores, donde se hace necesario emplear recursos y capacidades diferenciadas para impulsar no solo la creatividad sino también la innovación en sus diversas formas. Por tal razón, el objetivo es analizar el impacto de la gestión tecnológica sobre la capacidad de innovación en el sector restaurantero de Medellín, Colombia.

\section{Revisión de literatura}

\subsection{Gestión tecnológica}

El concepto de gestión tecnológica se ha consolidado como un sub-campo de la administración, cuyo objeto de estudio es la innovación (Pilkington y Teichert, 2006). Drejer (1997) identifica al menos cuatro momentos en la evolución del concepto, donde primero inició como una noción de investigación y desarrollo ( $\mathrm{I}+\mathrm{D})$, después se denominó planificación tecnológica y gestión de la innovación, hasta visualizarse como la dirección estratégica de la tecnología, usando formalmente el término de gestión tecnológica. Ya en el transcurso de los últimos años, la gestión tecnológica ha pasado de ser una práctica exclusiva del sector industrial a una también adoptada por el sector de los servicios (Badawy, 2009; Delgado et al., 2017a; Liao, 2005; McDermott, Kang y Walsh, 2001; Kang, 2006).
Este tipo de gestión integra los recursos tecnológicos de carácter duro (e.g. hardwares, máquinas, aparatos y equipos) y blando (e.g softwares, conocimientos, productos, procesos, sistemas y métodos) para la creación de bienes y la prestación de servicios. La tecnología se entiende como un recurso que resulta de los conocimientos tácitos y explícitos del ser humano que son útilmente aplicados en la solución de problemas (Kang, 2006; Phaal, Farrukh y Probert, 2004). Por consiguiente, la gestión tecnológica es la capacidad de las organizaciones para reconfigurar su base tecnológica e implementar objetivos centrados en la innovación a través de prácticas que mejoran significativamente los productos, los procesos y otros. A la par que la gestión tecnológica facilita la generación de conocimientos y competencias más complejas (Cetindamar et al., 2009; Huarng, 2010).

La gestión tecnológica implica tener un liderazgo y una visión abierta del uso de recursos y capacidades tecnológicas para soportar la creación, el desarrollo y la comercialización de las innovaciones (Medcof y Lee, 2017). Las empresas deben identificar mecanismos estratégicos para la asimilación de tecnologías y la integración de actividades de $\mathrm{I}+\mathrm{D}$ que incidan en el fortalecimiento de su competitividad y posicionamiento en el mercado (Krawczyk-Dembicka, 2017).

Entre estas actividades se enlista la estrategia tecnológica, la cual está relacionada con la identificación de oportunidades y amenazas para la toma de decisiones con base en la I+D y la planeación de tecnologías (AranaSolares, Ortega-Jiménez, Alfalla-Luque y Pérez-Díez de los Ríos, 2018). Esta tarea circunscribe procesos de búsqueda y compilación de información sobre el entorno, que ayudan a detectar el valor de las tecnologías en el desarrollo de innovaciones (Cetindamar et al., 2016a). En este sentido, la estrategia tecnológica provee una guía para la gestión empresarial, asegurando el alineamiento entre los objetivos organizacionales y las actividades tecnológicas (Panda y Sharma, 2020). Por tal razón, se incluyen estrategias que posibilitan mejorar la relación con los clientes y la prestación de servicios mediante las tecnologías. Otro elemento estratégico se refiere a la protección del patrimonio tecnológico, tarea que considera la implantación de políticas y el uso de instrumentos legales para asegurar que los activos intelectuales agregados en las innovaciones no sean hurtados y utilizados sin consentimiento (Cetindamar et al., 2009; 2016a; Panda y Sharma, 2020; Vargas-Sánchez y LópezGuzmán 2018).

Por otro lado, las prácticas de I+D soportan directamente el progreso y perfeccionamiento de las innovaciones. Por ende, es indispensable tener el suficiente financiamiento que constituye una base para lanzar nuevas ideas, ampliar los conocimientos existentes y ejecutar los proyectos de investigación (Berbegal-Mirabent, De La Torre y Gil- 
Doménech, 2020; Tou, Watanabe y Neittaanmäki, 2019; Wonglimpiyarat, 2019). Asimismo, estas prácticas dependen de la parte comercial, donde la rapidez en la que se lanza un nuevo bien, servicio o proceso en el mercado, convirtiéndose así, en un aspecto de la gestión tecnológica (Phaal et al., 2006).

Por su parte, la asimilación tecnológica da cuenta de qué tanto la empresa explota la tecnología para innovar (Delgado et al., 2017a). En esta actividad se suman la adquisición y el aprovechamiento de las tecnologías, así como las habilidades y la capacitación en el manejo tecnológico. Por su parte, la adquisición se refiere a cómo la empresa invierte en tecnologías valiosas, las cuales pueden ser desarrolladas internamente a través de alguna forma de colaboración o adquiridas de externos. Una vez que se ha invertido en tecnología, la empresa debe ser capaz de asimilarlas para su beneficio y, requiere de mecanismos de adopción y adaptación. Vinculado con esto, se encuentran las habilidades de los trabajadores ya que sin éstas las tecnologías podrían convertirse en obsoletas al no generar resultados positivos. En consecuencia, la capacitación se hace parte crucial en el desarrollo de la competencia tecnológica del personal y de la misma empresa (Cetindamar et al., 2016a).

\subsection{Capacidad de innovación}

La innovación se puede concebir como un resultado y una capacidad. En cuanto a la primera concepción, se trata de un nuevo o mejorado producto (bien o servicio), proceso, método de producción, fuente de materia prima o la reorganización de todo un sector, industria o empresa (Damanpour, Walker y Avellaneda, 2009; Schumpeter, 1934). Mientras que como capacidad se refiere a la habilidad de absorber, combinar y transformar recursos organizacionales para generar valor por encima del ya conocido en el mercado (Delgado et al., 2018). Asimismo, esta capacidad enfatiza el papel clave de la dirección estratégica para adaptar, integrar y reconfigurar de manera apropiada los recursos tanto internos como externos frente a las contingencias del entorno (Ravichandran, 2018).

La capacidad de innovación implica una visión renovada con aristas hacia el aumento de la competitividad, la creación de valor y la consolidación de una cultura centrada en los clientes y colaboradores (Anning-Dorson, 2017; Delgado, Vargas, Rodríguez y Montes 2017b). De tal manera que el descubrimiento y aprovechamiento de las oportunidades en el sector junto con los aspectos organizacionales, humanos y relacionales permitirá desplegar la capacidad de innovación y, con ello, nuevas propuestas de valor (Szeto, 2000; Tajvidi y Karami, 2015). La literatura da pauta para considerar diversos aspectos, entre ellos: la estructura organizacional, el capital humano y las redes de colaboración.

En este sentido, la estructura organizacional es la forma interna de una entidad empresarial que apoya en dar respuesta rápida y eficiente a los procesos de gestión (De Mello, Marx y Salerno, 2012). La estructura se soporta de la cultura organizacional y la toma de decisiones (Dedahanov, Rhee y Yoon, 2017) para facilitar el trabajo integrado, la comunicación y un ambiente imprescindibles en el desarrollo de innovaciones (De Mello et al., 2012). A nivel estratégico, la dirección y el liderazgo permiten interpretar de manera adecuada el entorno para que la empresa pueda adaptarse y saber cómo actuar ante los cambios. Al mismo tiempo, de desenvolver conocimientos en el desarrollo de nuevos productos y procesos, así como prácticas organizacionales y de comercialización (Delgado, Vargas, Montes y Rodríguez, 2016; Kalkan, Bozkurt y Arman, 2014).

Por su parte, el capital humano como componente de la capacidad de innovación es constituido por las habilidades intelectuales de las personas que producen valor a la empresa (Fonseca, de Faria y Lima, 2019; Kianto, Sáenz y Aramburu, 2017). Aspecto que involucran la inversión para proporcionar conocimientos y formación a las personas, permitiéndoles desarrollar sus destrezas productivas y creativas (Delgado et al., 2018). De igual manera, el nivel educativo de los trabajadores se considera un indicador, existiendo mejores oportunidades de innovación cuando se cuenta con personas más calificadas (Fonseca et al., 2018; Sun, Li y Ghosal, 2020; Pasban y Nojedeh, 2016). Otro factor es la gestión del conocimiento, cuyas acciones están destinadas a sistematizar los procesos de creación, almacenamiento y transformación de los conocimientos que cimientan la capacidad de innovación (Delgado et al., 2017b; Kianto et al., 2017). En consecuencia, el conocimiento debe ser documentado y compartido con los miembros de la empresa, con el fin de que exista un aprendizaje para estimular la innovación (McGuirk, Lenihan y Hart, 2015).

Junto con los recursos organizacionales y provenientes de las personas, también se suman los de carácter relacional o interactivo (Delgado et al., 2018). Por ejemplo, las redes de colaboración son una de las principales vías para acceder a la experiencia de otras entidades en sus actividades de innovación (Berbegal-Mirabent et al., 2020; Bouncken, Fredrich, Kraus y Ritala, 2020; Delgado et al., 2018; Hohberger, Kruger y Almeida, 2020). De manera que estas redes forjan alianzas estratégicas y acuerdos de cooperación con otros actores que se involucran en las tareas de innovación, útiles para optimizar los procesos de abastecimiento, obtener nuevos conocimientos y mejorar el posicionamiento de los involucrados (Bicen, Hunt y Madhavaram, 2021; Bouncken, Ratzmann, Pesch y Laudien, 2018; Hameed, Nisar y Wu, 2021; Zahoor y Al-Tabbaa, 2020). Desde esta perspectiva, las redes colaborativas generan un stock de conocimiento compartido que se va nutriendo de los flujos de información sobre los procedimientos, rutinas y sistemas tecnológicos que se producen entre los participantes (Hohberger et al., 2020). 


\subsection{Gestión tecnológica y capacidad de innovación en restaurantes}

Los restaurantes participan en un sector empresarial altamente volátil al sufrir constantemente de elevados niveles de incertidumbre y tasas de fracaso, necesitando de estrategias y prácticas vinculadas con la innovación. Ante tal situación, los operadores de restaurantes están utilizando cada vez más la tecnología para desarrollar nuevos productos, controlar sus actividades de manera más eficiente, proporcionar una mejor experiencia de servicio a los clientes y ampliar sus esfuerzos de comercialización; apuntando con ello, una fuerte vinculación entre la gestión tecnológica y la capacidad de innovación (Delgado et al., 2017b).

En este tipo de empresas, las aplicaciones de la tecnología son múltiples y variadas que van desde el uso de equipo de cómputo e Internet para actividades administrativas, hasta sistemas de información, máquinas y equipos novedosos inmersos en la producción de alimentos y el servicio ante el comensal (Delgado et al., 2016; Hjalager, 1999; Heo, 2016; Hurtado, Jaría-Chacón y Valls-Pasola, 2017; Ruiz et al., 2014). A tal grado que el liderazgo en gestión tecnológica (Medcof y Lee, 2017) y prácticas de I+D son motores para la generación de innovaciones (Tajvidi y Karami, 2015).

La innovación en el contexto de los restaurantes adquiere mayor significado, cuando se traspasan las concepciones tradicionales del desarrollo económico hacia nuevos paradigmas basados en el valor de los intangibles. En este tenor, Penrose (1959), Barney (1991), Grant (1991) y otros precursores de la visión basada en recursos, manifestaron que la competitividad dependía en gran medida de cómo las empresas gestionan sus recursos a través de capacidades, logrando combinaciones únicas, valiosas, inimitables e insustituibles. Enfatizando con esto, el protagonismo de los recursos intangibles al ser más difíciles de gestionar, medir y contabilizar como activos.

Por su parte, la teoría de las capacidades dinámicas incorpora los supuestos de la visión basada en recursos y la parte del entorno externo de la organización para potencializar sus ventajas competitivas (Teece, Pisano y Shuen, 1997; Wernerfelt, 1984). Así, las capacidades dinámicas se conciben como aquellas que utilizan las empresas para integrar, construir y reconfigurar las competencias internas y externas y, con éstas hacer frente a los cambios drásticos del entorno (Teece et al., 1997). Asimismo, este enfoque teórico explica que la naturaleza de la empresa no está determinada solo por aspectos tangibles y monetarios, sino que también por aspectos inmateriales que permiten entregar una mezcla única de valor (Teece y Leih, 2016; Teece, Peteraf y Leih, 2016).
Con base en estos aspectos teóricos, se entiende que los recursos tecnológicos pueden ser únicos e inimitables posibilitando la creación de ventajas competitivas. Por consiguiente, es necesario aplicar sobre tales recursos una capacidad compleja como la gestión tecnológica. De manera que la gestión tecnológica aprovecha los recursos internos y externos de los restaurantes para incidir sobre la capacidad de innovación (Delgado et al., 2017a; Delgado et al., 2016). En suma, la gestión tecnológica a través de la estrategia tecnológica, la asimilación tecnológica y las prácticas de $\mathrm{I}+\mathrm{D}$, se presenta como una oportunidad para direccionar los recursos organizacionales, humanos y colaborativos en el desarrollo de la capacidad de innovación. Por ende, se postula la siguiente hipótesis de investigación:

$\mathrm{Hc}=$ La gestión tecnológica tiene un impacto significativo y positivo sobre la capacidad de innovación en los restaurantes de Medellín.

\section{Metodología}

\subsection{Tipo de estudio}

El trabajo se fundamentó en el enfoque cuantitativo y el método hipotético-deductivo, al contrastar una explicación teórica en la realidad del sector restaurantero de Medellín, Colombia. Fue de diseño no experimental y de corte transversal al no existir un control deliberado sobre las variables en estudio y recolectar los datos en solo un momento, mostrando el fenómeno tal cual se presenta. Además, su alcance fue explicativo al determinar la incidencia de la gestión tecnológica sobre la capacidad de innovación de los restaurantes.

\subsection{Muestra y recolección de datos}

Se llevó a cabo un muestreo no probabilístico por criterio. Primero fue necesario identificar los restaurantes de la zona de estudio a partir de las guías de Tour Gastronómico (2019) y TripAdvisor (2020). Después se invitaron a 190 restaurantes de los cuales 139 participaron, es decir, $73.15 \%$. La técnica de recolección de datos fue la encuesta auto-administrada a los directivos y gerentes de cada empresa a través de correo electrónico y directamente en los establecimientos. Así, se trató de una muestra de micro, pequeñas y medianas empresas de reciente operación y jóvenes-maduras, dirigidas por socios, familias y únicos dueños. Además, de ser restaurantes con una vasta variedad gastronómica para atender las demandas de un mercado local con poder adquisitivo medio (Tabla 1 ). 
Tabla 1 Características de los restaurantes $(\mathrm{n}=139)$

\begin{tabular}{|c|c|c|c|}
\hline Variable & Valor & $\begin{array}{c}\text { Frecuencia } \\
(f)\end{array}$ & $\begin{array}{c}\text { Porcentaje } \\
(\%)\end{array}$ \\
\hline \multirow{3}{*}{$\begin{array}{c}\text { Tamaño de la } \\
\text { empresa }\end{array}$} & Micro (1-10 trabajadores) & 77 & 55.4 \\
\hline & Pequeña (10-50 trabajadores) & 40 & 28.8 \\
\hline & Mediana (51-100 trabajadores) & 22 & 15.8 \\
\hline \multirow{6}{*}{ Tipo de empresa } & Familia & 39 & 28.1 \\
\hline & Grupo de socios & 55 & 39.6 \\
\hline & Único dueño & 25 & 18 \\
\hline & Franquicia & 8 & 5.8 \\
\hline & Cadena & 7 & 5 \\
\hline & Otro & 5 & 3.6 \\
\hline \multirow{8}{*}{$\begin{array}{l}\text { Antigüedad de la } \\
\text { empresa }\end{array}$} & Menos de un año & 26 & 18.7 \\
\hline & $1-5$ años & 49 & 35.3 \\
\hline & 6-10 años & 20 & 14.4 \\
\hline & $11-15$ años & 15 & 10.8 \\
\hline & 16-20 años & 8 & 5.8 \\
\hline & $21-25$ años & 5 & 3.6 \\
\hline & 26-30 años & 5 & 3.6 \\
\hline & Más de 30 años & 11 & 7.9 \\
\hline \multirow{6}{*}{$\begin{array}{l}\text { Gasto promedio de } \\
\text { consumo del cliente }\end{array}$} & 2-10 dólares & 41 & 29.5 \\
\hline & 11-20 dólares & 68 & 48.9 \\
\hline & 21-30 dólares & 15 & 10.8 \\
\hline & 31-40 dólares & 11 & 7.9 \\
\hline & 41-50 dólares & 2 & 1.4 \\
\hline & 61-70 dólares & 2 & 1.4 \\
\hline \multirow{3}{*}{ Tipo de cliente } & Residente & 115 & 82.7 \\
\hline & Turista nacional & 4 & 2.9 \\
\hline & Turista extranjero & 20 & 14.4 \\
\hline \multirow{11}{*}{$\begin{array}{l}\text { Especialidad } \\
\text { culinaria }\end{array}$} & Colombiana & 19 & 13.7 \\
\hline & Mexicana & 3 & 2.2 \\
\hline & Del mar & 9 & 6.5 \\
\hline & Cortes de carne & 12 & 8.6 \\
\hline & Peruana & 9 & 6.5 \\
\hline & Americana & 9 & 6.5 \\
\hline & Italiana & 18 & 12.9 \\
\hline & Oriental & 5 & 3.6 \\
\hline & Fusión & 18 & 12.9 \\
\hline & Vegetariana & 4 & 2.9 \\
\hline & Otra & 33 & 23.7 \\
\hline
\end{tabular}

\subsection{Instrumento de medición}

El instrumento se construyó a partir de la revisión de literatura, opinión de expertos y una prueba piloto. El cuestionario final se integró por dos secciones. En la primera, se encontraron los 39 ítems para la apreciación de las variables con una escala tipo Likert de seis puntos que fue de 1 "totalmente en desacuerdo" a 6 "totalmente de acuerdo" (Tabla 2). La segunda sección se refirió a los datos de los establecimientos tales como años de operación, tamaño de la empresa, tipo de cliente, consumo promedio y especialidad culinaria (Tabla 1). 
Tabla 2 Operacionalización de las variables

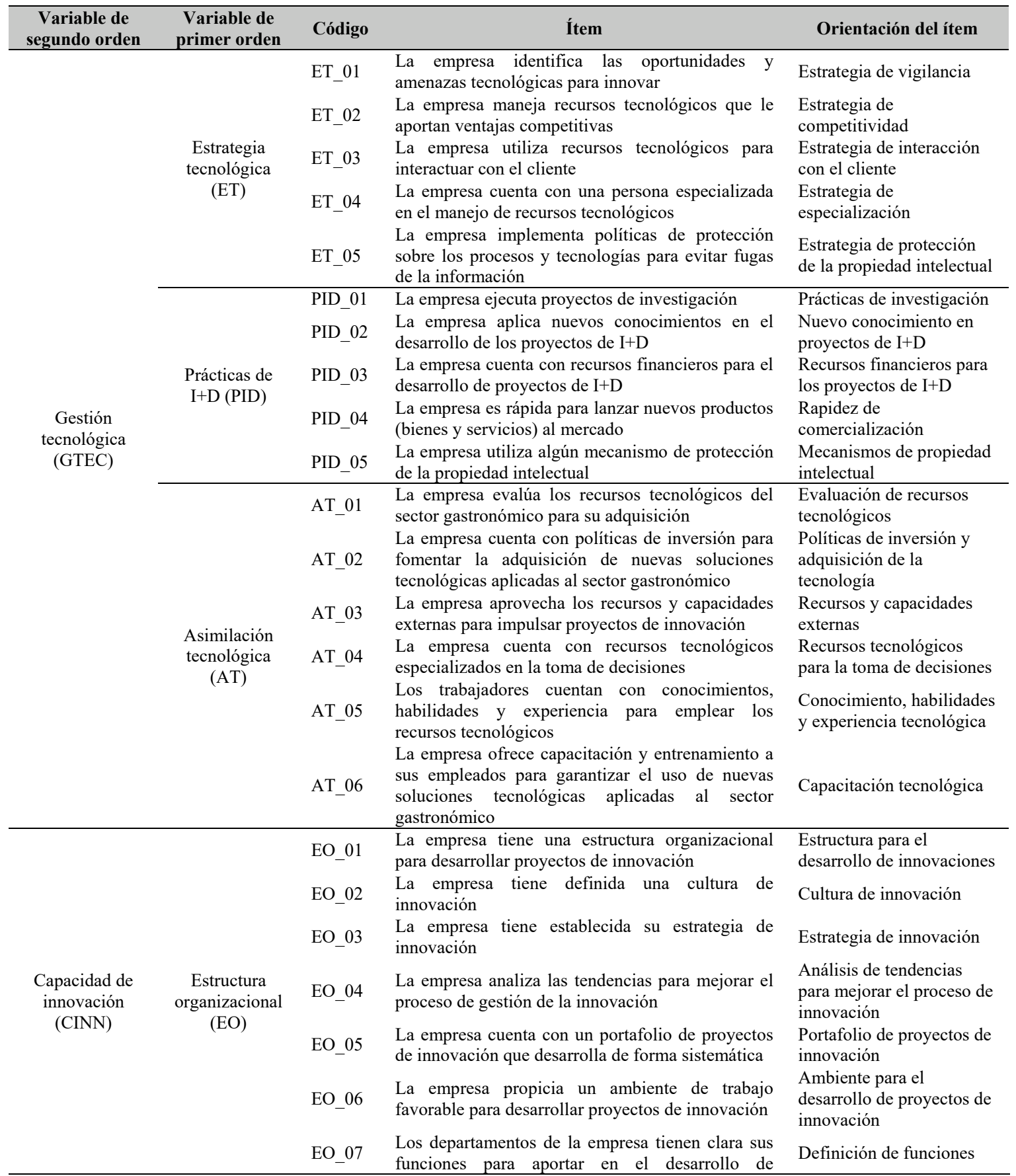




\begin{tabular}{|c|c|c|c|c|}
\hline $\begin{array}{c}\text { Variable de } \\
\text { segundo orden }\end{array}$ & $\begin{array}{l}\text { Variable de } \\
\text { primer orden }\end{array}$ & Código & Ítem & Orientación del ítem \\
\hline & & EO_08 & $\begin{array}{l}\text { proyectos de innovación } \\
\text { Existe el trabajo integrado entre los departamentos } \\
\text { para el desarrollo de innovaciones }\end{array}$ & Trabajo integrado \\
\hline & & EO_09 & $\begin{array}{l}\text { La empresa brinda la oportunidad de que todos los } \\
\text { miembros desarrollen su habilidad de liderazgo } \\
\text { para la generación de proyectos de innovación }\end{array}$ & Liderazgo \\
\hline & & EO_10 & $\begin{array}{l}\text { Existe libertad en la toma de decisiones de los } \\
\text { trabajadores para fomentar proyectos de } \\
\text { innovación }\end{array}$ & Toma de decisiones \\
\hline & & EO_11 & $\begin{array}{l}\text { Existe comunicación eficaz para que los } \\
\text { trabajadores puedan aportar ideas que fomenten } \\
\text { proyectos de innovación }\end{array}$ & Comunicación eficaz \\
\hline & \multirow{8}{*}{$\begin{array}{c}\text { Capital } \\
\text { humano }(\mathrm{CH})\end{array}$} & CH_01 & $\begin{array}{l}\text { La empresa ofrece entrenamiento o capacitación } \\
\text { especializada a sus trabajadores para generar } \\
\text { nuevos productos (bienes y servicios) }\end{array}$ & $\begin{array}{l}\text { Capacitación del recurso } \\
\text { humano }\end{array}$ \\
\hline & & CH_02 & $\begin{array}{l}\text { La empresa contrata a los mejores recursos } \\
\text { humanos del sector gastronómico }\end{array}$ & $\begin{array}{l}\text { Cualificación del recurso } \\
\text { humano }\end{array}$ \\
\hline & & CH_03 & $\begin{array}{l}\text { Los trabajadores se muestran motivados para } \\
\text { generar ideas creativas y novedosas }\end{array}$ & $\begin{array}{l}\text { Motivación para la } \\
\text { generación de ideas }\end{array}$ \\
\hline & & CH_04 & $\begin{array}{l}\text { La empresa tiene un sistema de recompensas y } \\
\text { reconocimiento para los trabajadores }\end{array}$ & $\begin{array}{l}\text { Recompensas y } \\
\text { reconocimiento }\end{array}$ \\
\hline & & CH_05 & $\begin{array}{l}\text { La empresa gestiona el conocimiento para el } \\
\text { beneficio de los trabajadores }\end{array}$ & Conocimiento compartido \\
\hline & & CH_06 & $\begin{array}{l}\text { Existe intercambio de conocimiento entre los } \\
\text { trabajadores }\end{array}$ & $\begin{array}{l}\text { Conocimiento } \\
\text { intercambiado }\end{array}$ \\
\hline & & CH_07 & La empresa documenta el conocimiento generado & $\begin{array}{l}\text { Conocimiento } \\
\text { documentado }\end{array}$ \\
\hline & & CH_08 & $\begin{array}{l}\text { La empresa tiene una estrategia para incluir la } \\
\text { opinión del cliente en el desarrollo de nuevos o } \\
\text { mejores productos (bienes y servicios) }\end{array}$ & Conocimiento del cliente \\
\hline & \multirow{4}{*}{$\begin{array}{l}\text { Redes de } \\
\text { colaboración } \\
\quad(\mathrm{RC})\end{array}$} & RC_01 & $\begin{array}{l}\text { La empresa tiene una estrategia para trabajar de } \\
\text { manera conjunta con los proveedores con el fin de } \\
\text { mejorar sus procesos de abastecimiento }\end{array}$ & $\begin{array}{l}\text { Colaboración con } \\
\text { proveedores }\end{array}$ \\
\hline & & RC_02 & $\begin{array}{l}\text { La empresa ha creado alianzas estratégicas con } \\
\text { otras empresas del sector turístico para aumentar } \\
\text { su posicionamiento en el mercado }\end{array}$ & $\begin{array}{l}\text { Colaboración con } \\
\text { empresas turísticas }\end{array}$ \\
\hline & & RC_03 & $\begin{array}{l}\text { La empresa tiene relaciones con los centros de } \\
\text { investigación o universidades con el fin de } \\
\text { desarrollar proyectos de innovación }\end{array}$ & $\begin{array}{l}\text { Colaboración con centros } \\
\text { de investigación y } \\
\text { universidades }\end{array}$ \\
\hline & & RC_04 & $\begin{array}{l}\text { La empresa se vincula con organizaciones } \\
\text { gubernamentales con el propósito de aumentar su } \\
\text { participación en el mercado }\end{array}$ & $\begin{array}{l}\text { Colaboración con } \\
\text { dependencias } \\
\text { gubernamentales }\end{array}$ \\
\hline
\end{tabular}

\subsection{Estimación estadística}

Para estudiar el impacto de la gestión tecnológica sobre la capacidad de innovación se recurrió a la modelación de ecuaciones estructurales por mínimos cuadrados parciales (PLS-SEM, por sus siglas en inglés), en razón de tres consideraciones. Primero, por tratarse de un número muestral pequeño de acuerdo con los criterios estadísticos de representatividad; segundo, supone un enfoque de modelado suave sobre la distribución de datos; y tercero, porque las aplicaciones tienen poca teoría disponible y la precisión predictiva es primordial para el estudio (Hair, Hult, Ringle y Sarstedt, 2017; Hwang, Malhotra, Kim, Tomiuk y Hong, 2010). En este sentido, fue necesario evaluar tanto el modelo de medida como el estructural para realizar las interpretaciones. Asimismo, se emplearon las medidas de tendencia central y la correlación bivariada de Spearman. En el tratamiento de los datos se utilizaron los softwares Smart PLS versión 3.3.3 (Ringle, Wende y Becker, 2015) y SPSS versión 26.0 (IBM, 2019).

\section{Resultados}

\subsection{Modelo de medida}

Para la evaluación del modelo de medida se cotejó la consistencia interna a través del alfa de Cronbach $(\alpha)$, el rho_A y la fiabilidad compuesta $\left(\rho_{\mathrm{c}}\right)$. Como se observa en la Tabla 3, los valores son satisfactorios $(\alpha>0.700$; rho $\mathrm{A}>0.700 ; \rho_{\mathrm{c}}>0.700$ ) (Henseler, Ringle y Sinkovics, 2009; Nunnally y Bernstein, 1994). Se cumplió con la validez convergente y discriminante (Tabla 3 ), puesto que la varianza media extraída (AVE, por sus siglas en inglés) fue mayor a 0.500 en todos los casos y la raíz cuadrada de la AVE fue superior a la correlación entre variables (Fornell y Larcker, 1981). Asimismo, se corroboró la ratio heterotrait-monotrail (HTMT), valores que fueron inferiores a 0.900 , tal como se recomienda (Henseler, Ringle y Sartedt, 2016). 
Tabla 3 Confiabilidad, validez convergente y discriminante

*Raíz cuadrada de la varianza media extraida (AVE)

\begin{tabular}{|c|c|c|c|c|c|c|c|c|c|c|c|}
\hline \multirow{2}{*}{$\begin{array}{l}\text { Variable } \\
\text { de } \\
\text { segundo } \\
\text { orden }\end{array}$} & \multirow{2}{*}{$\begin{array}{c}\text { Variable } \\
\text { de primer } \\
\text { orden }\end{array}$} & \multirow[b]{2}{*}{$\alpha$} & \multirow[b]{2}{*}{ rho_A } & \multirow[b]{2}{*}{$\rho_{c}$} & \multirow[b]{2}{*}{ AVE } & \multicolumn{6}{|c|}{ Correlaciones } \\
\hline & & & & & & ET & PID & AT & EO & $\mathbf{C H}$ & $\mathbf{R C}$ \\
\hline \multirow{3}{*}{ GTEC } & ET & 0.829 & 0.830 & 0.880 & 0.595 & $0.771^{*}$ & & & & & \\
\hline & PID & 0.914 & 0.922 & 0.936 & 0.747 & 0.652 & $0.864^{*}$ & & & & \\
\hline & AT & 0.895 & 0.897 & 0.920 & 0.657 & 0.643 & 0.739 & $0.811^{*}$ & & & \\
\hline \multirow{3}{*}{ CINN } & EO & 0.958 & 0.959 & 0.963 & 0.704 & 0.536 & 0.598 & 0.800 & $0.839^{*}$ & & \\
\hline & $\mathrm{CH}$ & 0.904 & 0.907 & 0.923 & 0.600 & 0.536 & 0.533 & 0.661 & 0.760 & $0.774^{*}$ & \\
\hline & $\mathrm{RC}$ & 0.757 & 0.764 & 0.845 & 0.577 & 0.504 & 0.503 & 0.618 & 0.609 & 0.592 & $0.760^{*}$ \\
\hline
\end{tabular}

La Tabla 4 expone las cargas cruzadas de cada ítem, mostrando correlaciones simples de las medidas con su respectivo constructo, como se aprecia los valores son mayores al criterio mínimo recomendado $(>0.707)$, soportando con ello, la pertinencia de las mediciones (Hair et al., 2017; Henseler et al., 2009).

Tabla 4 Cargas cruzadas

\begin{tabular}{|c|c|c|c|c|c|c|}
\hline Ítem & $\begin{array}{c}\text { Estrategia } \\
\text { tecnológica } \\
\text { (ET) }\end{array}$ & $\begin{array}{c}\text { Prácticas de } \\
\text { investigación y } \\
\text { desarrollo } \\
\text { (PID) }\end{array}$ & $\begin{array}{c}\text { Asimilación } \\
\text { tecnológica } \\
\text { (AT) }\end{array}$ & $\begin{array}{c}\text { Estructura } \\
\text { organizacional } \\
\text { (EO) }\end{array}$ & $\begin{array}{c}\text { Capital } \\
\text { humano }(\mathbf{C H})\end{array}$ & $\begin{array}{c}\text { Redes de } \\
\text { colaboración } \\
\text { (RC) }\end{array}$ \\
\hline ET_01 & 0.842 & 0.527 & 0.535 & 0.505 & 0.409 & 0.429 \\
\hline ET_02 & 0.792 & 0.442 & 0.477 & 0.298 & 0.382 & 0.398 \\
\hline ET_03 & 0.759 & 0.473 & 0.460 & 0.375 & 0.396 & 0.308 \\
\hline ET_04 & 0.747 & 0.507 & 0.495 & 0.422 & 0.333 & 0.330 \\
\hline ET_05 & 0.711 & 0.558 & 0.505 & 0.452 & 0.541 & 0.468 \\
\hline PID_01 & 0.575 & 0.904 & 0.717 & 0.609 & 0.506 & 0.462 \\
\hline PID_02 & 0.615 & 0.937 & 0.731 & 0.635 & 0.538 & 0.480 \\
\hline PID_03 & 0.533 & 0.892 & 0.633 & 0.518 & 0.409 & 0.440 \\
\hline PID_04 & 0.531 & 0.791 & 0.549 & 0.359 & 0.409 & 0.390 \\
\hline PID_05 & 0.562 & 0.786 & 0.541 & 0.427 & 0.431 & 0.394 \\
\hline AT_01 & 0.566 & 0.605 & 0.796 & 0.562 & 0.603 & 0.532 \\
\hline AT_02 & 0.542 & 0.664 & 0.862 & 0.628 & 0.545 & 0.501 \\
\hline AT_03 & 0.510 & 0.635 & 0.823 & 0.685 & 0.493 & 0.486 \\
\hline AT_04 & 0.589 & 0.619 & 0.775 & 0.664 & 0.482 & 0.488 \\
\hline AT_05 & 0.445 & 0.532 & 0.779 & 0.683 & 0.534 & 0.486 \\
\hline AT_06 & 0.464 & 0.527 & 0.826 & 0.678 & 0.561 & 0.515 \\
\hline EO_01 & 0.428 & 0.573 & 0.709 & 0.840 & 0.634 & 0.503 \\
\hline EO_02 & 0.389 & 0.500 & 0.584 & 0.789 & 0.611 & 0.429 \\
\hline EO_03 & 0.415 & 0.487 & 0.636 & 0.814 & 0.616 & 0.480 \\
\hline EO_04 & 0.512 & 0.533 & 0.676 & 0.812 & 0.581 & 0.521 \\
\hline EO_05 & 0.496 & 0.580 & 0.719 & 0.803 & 0.605 & 0.555 \\
\hline EO_06 & 0.458 & 0.465 & 0.697 & 0.848 & 0.592 & 0.541 \\
\hline EO_07 & 0.433 & 0.526 & 0.699 & 0.872 & 0.671 & 0.511 \\
\hline EO_08 & 0.485 & 0.477 & 0.663 & 0.886 & 0.665 & 0.510 \\
\hline EO_09 & 0.510 & 0.521 & 0.709 & 0.883 & 0.655 & 0.572 \\
\hline $\mathrm{EO}_{-}^{-} 10$ & 0.380 & 0.406 & 0.627 & 0.836 & 0.675 & 0.483 \\
\hline EO_-11 & 0.437 & 0.461 & 0.665 & 0.839 & 0.702 & 0.511 \\
\hline CH_01 & 0.417 & 0.350 & 0.599 & 0.669 & 0.792 & 0.505 \\
\hline CH_02 & 0.364 & 0.360 & 0.515 & 0.566 & 0.739 & 0.419 \\
\hline CH_03 & 0.431 & 0.483 & 0.567 & 0.657 & 0.837 & 0.437 \\
\hline CH_04 & 0.451 & 0.469 & 0.454 & 0.561 & 0.721 & 0.470 \\
\hline CH_05 & 0.500 & 0.454 & 0.551 & 0.642 & 0.839 & 0.497 \\
\hline CH_06 & 0.375 & 0.445 & 0.452 & 0.515 & 0.764 & 0.356 \\
\hline CH_07 & 0.461 & 0.517 & 0.530 & 0.583 & 0.767 & 0.516 \\
\hline CH_08 & 0.302 & 0.212 & 0.399 & 0.486 & 0.727 & 0.460 \\
\hline RC_01 & 0.417 & 0.370 & 0.466 & 0.480 & 0.638 & 0.785 \\
\hline $\mathrm{RC}_{-}^{-} 02$ & 0.324 & 0.303 & 0.503 & 0.444 & 0.401 & 0.739 \\
\hline RC_03 & 0.404 & 0.427 & 0.467 & 0.531 & 0.388 & 0.766 \\
\hline $\mathrm{RC}^{-} 04$ & 0.379 & 0.441 & 0.444 & 0.379 & 0.325 & 0.747 \\
\hline
\end{tabular}




\subsection{Modelo estructural}

Para garantizar el ajuste y validez del modelo estructural se utilizó la función bootstrapping con un total de 5,000 casos (Dijkstra y Henseler, 2015). La Tabla 5 muestra los valores de $t$ y significancia para cada uno de los coeficientes, evidenciando su significancia $(\mathrm{t} \geq 1.960 ; \mathrm{p}<0.001)$. De igual manera, al aplicar un análisis blindfolding el índice de redundancia de valor cruzado $\left(\mathrm{Q}^{2}\right)$ fue mayor a cero en todos los casos, manifestando la relevancia predictiva del modelo (Geisser, 1974; Stone, 1974).

Por otro lado, se presentó una buena bondad de ajuste al tener una raíz cuadrada media residual (SRMR, por sus siglas en inglés) de 0.077 (Hu y Bentler, 1999). Aunque los expertos señalan que este indicador puede ser flexible cuando las variables y sus relaciones todavía se están explorando (Hair et al., 2017).

Tabla 5 Coeficientes estandarizados y relevancia predictiva del modelo

\begin{tabular}{|c|c|c|c|c|c|c|c|c|}
\hline Dinámica & $\begin{array}{c}\text { Muestra } \\
\text { original }\end{array}$ & $\begin{array}{c}\text { Media de } \\
\text { la } \\
\text { muestra }\end{array}$ & $\begin{array}{c}\text { Desviación } \\
\text { estándar }\end{array}$ & Valor t & Sig. & $\mathbf{R}^{2}$ & $\mathbf{R}_{a j}^{2}$ & $\mathbf{Q}^{2}$ \\
\hline GTEC $\rightarrow$ CINN & 0.771 & 0.770 & 0.038 & 20.368 & 0.000 & 0.594 & 0.591 & 0.281 \\
\hline GTEC $\rightarrow$ PID & 0.907 & 0.908 & 0.019 & 47.846 & 0.000 & 0.822 & 0.821 & 0.572 \\
\hline $\mathrm{GTEC} \rightarrow \mathrm{AT}$ & 0.916 & 0.917 & 0.013 & 72.382 & 0.000 & 0.839 & 0.837 & 0.507 \\
\hline $\mathrm{CINN} \rightarrow \mathrm{EO}$ & 0.949 & 0.950 & 0.013 & 75.583 & 0.000 & 0.901 & 0.900 & 0.588 \\
\hline
\end{tabular}

\subsection{Interpretación del modelo}

En la Figura 1, se puede observar que la trayectoria estructural de la gestión tecnológica sobre la capacidad de innovación es positiva y significativa $(\beta=0.771 ; \mathrm{p} \leq 0.001)$. El coeficiente de determinación $\left(\mathrm{R}^{2}\right)$ de 0.591 significa que $51 \%$ de la varianza de la capacidad de innovación está explicada por la variable independiente. Además, la gestión tecnológica tiene una relevancia predictiva sobre la capacidad de innovación moderada-fuerte $\left(Q^{2}=0.281\right)$ (Hair et al., 2017). En Tabla 6 se aprecian los efectos indirectos que tiene la gestión tecnológica sobre las dimensiones de la capacidad de innovación, siendo el más alto con la estructura organizacional $(\beta=0.697 ; \mathrm{p} \leq 0.001)$, seguido del capital humano $(\beta=0.731 ; p \leq 0.001)$, y finalmente, con las redes de colaboración $(\beta=0.567 ; \mathrm{p} \leq 0.001)$. Estos resultados en conjunto dan soporte a la hipótesis central del trabajo $(\mathrm{Hc})$.
Figura 1 Modelo estructural del impacto de la gestión tecnológica sobre la capacidad de innovación

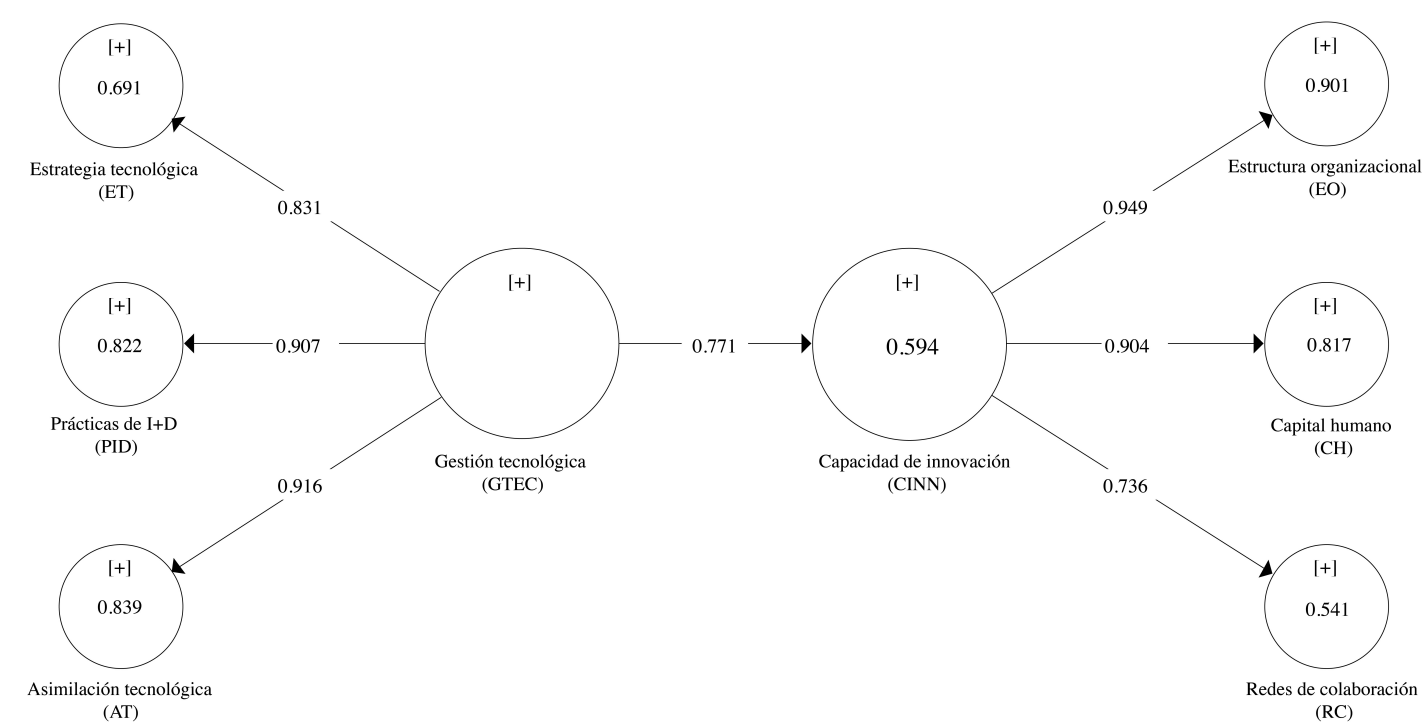


Tabla 6 Efectos indirectos de la gestión tecnológica

\begin{tabular}{ccccccc}
\hline Dinámica & $\begin{array}{c}\text { Efecto } \\
\text { indirecto }\end{array}$ & $\begin{array}{c}\text { Muestra } \\
\text { original }\end{array}$ & $\begin{array}{c}\text { Media de la } \\
\text { muestra }\end{array}$ & $\begin{array}{c}\text { Desviación } \\
\text { estándar }\end{array}$ & Valor t \\
\hline GTEC $\rightarrow$ EO & 0.731 & 0.731 & 0.732 & 0.039 & 18.756 \\
GTEC $\rightarrow$ CH & 0.697 & 0.697 & 0.696 & 0.045 & 15.522 \\
GTEC $\rightarrow$ RC & 0.567 & 0.567 & 0.570 & 0.043 & 13.236 & 0.000 \\
\hline
\end{tabular}

En apoyo a la interpretación del modelo se complementaron los resultados con los estadísticos descriptivos y la correlación de Spearman. En la Tabla 7 se observa que la estrategia $(\tilde{\mathrm{x}}=4.48 ; \sigma=0.90)$ y la asimilación tecnológica $(\tilde{\mathrm{x}}=4.525 ; \sigma=1.023)$ fueron evaluadas como positivas-bajas, mientras que las prácticas de $\mathrm{I}+\mathrm{D}$ tuvieron un puntaje negativo ( $\tilde{\mathrm{x}}=3.805 ; \sigma=1.263$ ). Por otro lado, el capital humano fue la variable mejor evaluada $(\tilde{x}=5.004 ; \sigma=0.822)$, indicando una fortaleza del sector restaurantero de Medellín. Empero, las redes de colaboración $(\tilde{\mathrm{x}}=4.057$; $\sigma=1.059)$ y la estructura organizacional $(\tilde{x}=4.779 ; \sigma=1.058)$ tuvieron un menor puntaje.
Tabla 7 Estadísticos descriptivos y correlaciones de Spearman

** La correlación es altamente significativa $(p \leq 0.010)$.

\begin{tabular}{cccccccccccc}
\hline $\begin{array}{c}\text { Variable de Variable de } \\
\text { segundo } \\
\text { orden }\end{array}$ & $\begin{array}{c}\text { primer } \\
\text { orden }\end{array}$ & Asimetría & Curtosis & Media & $\begin{array}{c}\text { Desviación } \\
\text { estándar }\end{array}$ & ET & PID & AT & EO & CH & RC \\
\hline \multirow{2}{*}{ GTEC } & ET & -0.538 & 0.314 & 4.486 & 0.909 & 1 & & & & \\
& PID & -0.128 & -0.855 & 3.805 & 1.263 & $0.671^{* *}$ & 1 & & & \\
& AT & -0.897 & 0.730 & 4.525 & 1.023 & $0.625^{* *}$ & $0.769^{* *}$ & 1 & & \\
CINN & EO & -1.359 & 1.562 & 4.779 & 1.058 & $0.499^{* *}$ & $0.655^{* *}$ & $0.776^{* *}$ & 1 & \\
& CH & -1.568 & 3.258 & 5.004 & 0.822 & $0.456^{* *}$ & $0.551^{* *}$ & $0.574^{* *}$ & $0.746^{* *}$ & 1 \\
& RC & -0.165 & -0.582 & 4.057 & 1.059 & $0.471^{* *}$ & $0.517^{* *}$ & $0.584^{* *}$ & $0.629^{* *}$ & $0.556^{* *}$ & 1 \\
\hline
\end{tabular}

Por otro lado, las correlaciones entre variables tuvieron fuerzas moderadas y fuertes (Tabla 6). En particular, las asociaciones de la estrategia tecnológica con la estructura organizacional $(\mathrm{r}=0.499 ; \mathrm{p} \leq 0.010)$, el capital humano $(\mathrm{r}=0.456 ; \mathrm{p} \leq 0.010)$ y las redes de colaboración $(\mathrm{r}=0.471$; $\mathrm{p} \leq 0.010$ ) fueron moderadas. Por tanto, al definir estrategias de vigilancia y especialización tecnológica, así como de interacción con el cliente y protección de la propiedad intelectual, se facilitarán las actividades de innovación, sobre todo aquellas conexas con el entrenamiento de los trabajadores y el conocimiento gestionado dentro de los restaurantes.

Las relaciones entre las prácticas de $\mathrm{I}+\mathrm{D}$ con la estructura organizacional $(\mathrm{r}=0.655 ; \mathrm{p} \leq 0.010)$, el capital humano $(\mathrm{r}=0.551 ; \mathrm{p} \leq 0.010)$ y las redes de colaboración $(\mathrm{r}=0.517$; $\mathrm{p} \leq 0.010)$ también fueron moderadas. En este tenor, la aplicación de nuevos conocimientos, los mecanismos de propiedad intelectual $\mathrm{y}$, las capacidades de financiamiento y comercialización de los proyectos de $\mathrm{I}+\mathrm{D}$, son aspectos vinculados con la estructura organizacional. En consecuencia, cuando la estructura organizacional se soporta por una estrategia y cultura orientadas en la innovación, se proveerá de un ambiente de liderazgo, toma de decisiones y comunicación para integrar el trabajo de las diferentes áreas de los restaurantes.

En la asimilación tecnológica se encuentra la relación más fuerte del estudio con la estructura organizacional $(\mathrm{r}=0.776$; $\mathrm{p} \leq 0.010$ ). Se puede decir que el sistema y la funcionalidad organizacional de los restaurantes con un marco de políticas, evaluaciones y competencias de carácter tecnológico son más propicios para la generación de innovaciones. Respecto a la cooperación con otros actores $(\mathrm{r}=0.584 ; \mathrm{p} \leq 0.010)$, se observa una relación cuando el arsenal tecnológico de la empresa es nutrido con recursos y capacidades externos. Mientras que el capital humano $(\mathrm{r}=0.574 ; \mathrm{p} \leq 0.010)$ puede verse favorecido cuando existe capacitación en el personal y, con ello, se detonan mejores conocimientos, habilidades y experiencia tecnológica.

\section{Discusión}

A diferencia de las aportaciones ya existentes sobre el uso de tecnológicas en restaurantes (Ahn y Seo, 2018; Heo, 2016; Hjalager, 1999; Kim et al., 2020; Moreno y Tejada, 2019; Leung y Loo, 2020; Park et al., 2021; Ruiz et al., 2014; Seyitoğlu e Ivanov, 2020), este trabajo desde la postura teórica de las capacidades dinámicas (Teece y Leih, 2016; Teece et al., 2016) evidencia que al tener una estrategia definida, llevar a cabo prácticas de I+D y asimilar eficazmente los recursos tecnológicos impactan positivamente sobre la capacidad de innovación de los restaurantes en los aspectos organizacionales, humanos y relacionales (Cetindamar et al., 2016a; Delgado et al., 2018; Damanpour et al., 2009; Huang et al., 2016; Ravichandran, 2018). 
A partir de esto, los resultados convergen con KrawczykDembicka (2017), Medcof y Lee (2017) y Arana-Solares et al. (2018), puesto que el liderazgo tecnológico permite identificar más fácilmente las amenazas que puedan mermar el desarrollo de innovaciones, el posicionamiento y la competitividad. Empero, el diagnóstico en los restaurantes de Medellín muestra como debilidad las prácticas de I+D al carecer de actividades de investigación, apoyos financieros $\mathrm{y}$ de un rápido lanzamiento de productos. Este hecho, puede incidir en cómo y con cuánta velocidad el sector restaurantero puede atender las demandas del mercado mediante sus innovaciones (Berbegal-Mirabent et al., 2020; Phaal et al., 2006; Tou et al., 2019; Wonglimpiyarat, 2019).

Por otro lado, destaca el capital humano como un aspecto positivo y fuerte en el sector de Medellín para generar innovaciones. Al igual que Pasban y Nojedeh (2016), Fonseca et al. (2018) y Sun et al. (2020), el componente intelectual de las personas genera valor en el desarrollo de nuevos bienes y servicios, así como introducir mejoras en las prácticas y actividades organizacionales. Como se apreció el capital humano está más relacionado con la asimilación tecnológica, ya que cuando existe capacitación se van enriqueciendo los saberes y el aprendizaje de las personas (Cetindamar et al., 2016a; Fonseca et al., 2019; Kianto et al., 2017). Aunque la asimilación tecnológica fue el aspecto mejor evaluado por los restaurantes se encuentra en un nivel bajo, por lo cual sería necesario replantarse cómo invertir y adquirir en nuevas tecnologías e, impulsar los proyectos de innovación con el apoyo de actores externos, tal como se señala en la literatura (Berbegal-Mirabent et al., 2020; Bouncken et al., 2020; Delgado et al., 2018; Hohberger et al. 2020)

Por último, una característica de las capacidades dinámicas es el uso de recursos externos (Teece y Leih, 2016; Teece et al., 1997) y, para lograrlo, es indispensable generar y mantener alianzas con otros actores. Respecto a las redes de colaboración, éstas fueron un aspecto valorado como positivo y bajo en comparación con las otras variables. Para contrarrestar esta situación es necesario trabajar con la estructura organizacional y la asimilación al vincularse directamente con el trabajo cooperativo que se puede tener con los proveedores, los centros de investigación, las universidades, las dependencias de gobierno y las empresas turísticas. En convergencia con Bouncken et al. (2018), Hohberger et al. (2020), Zahoor y Al-Tabbaa (2020), Bicen et al. (2021) y Hameed et al. (2021), los acuerdos de colaboración aumentan la efectividad de las actividades y los procesos de innovación.

\section{Conclusiones}

Los restaurantes son una fuerza impulsora de la economía de varios países, pero solo a través de la investigación de calidad se agregará valor para el desarrollo de su innovación; en especial, cuando va en aumento el uso de tecnologías en la operación y el servicio de los alimentos. En este tenor, el presente trabajo cumple satisfactoriamente su objetivo al determinar el poder explicativo de la gestión tecnológica sobre la capacidad de innovación en empresas restauranteras colombianas. Los hallazgos más destacados exponen que:

a) Las estrategias de vigilancia, competitividad y especialización tecnológica favorecen el aprendizaje y los conocimientos de las personas para llevar a cabo actividades de innovación.

b) La aplicación de nuevos conocimientos junto con las capacidades de financiamiento y comercialización para el desarrollo de proyectos de I+D, se relacionan con el sistema organizacional soportado por una cultura centrada en la innovación.

c) La asimilación de los recursos tecnológicos internos y externos de los restaurantes se asocian con la estrategia de innovación y, con ello, se potencian el liderazgo, la comunicación y el trabajo integrado.

d) La asimilación de recursos y capacidades tecnológicas está relacionada con las redes de colaboración que se tienen con otros actores (i.e. proveedores, centros de investigación, universidades, dependencias de gobierno y empresas turísticas), logrando fortalecer los conocimientos, las habilidades y la experiencia de los restaurantes.

Estos resultados son una contribución en la generación de nuevo conocimiento en cuanto a los vacíos detectados por otros investigadores respecto al análisis de la tecnología e innovación en restaurantes (Heo, 2016; Hjalager, 1999; Ruiz et al., 2014) y empresas de servicios (Badawy, 2009; Delgado et al., 2016; Liao, 2005; McDermott et al., 2001; Kang, 2006). Asimismo, se reconoce que el trabajo no es concluyente, ya que la gestión tecnológica y la capacidad de innovación tienen un extenso campo $\mathrm{y}$, por tanto, las dimensiones podrían abordarse desde otras visiones (Cetindamar et al., 2009; 2016a). No obstante, resulta ser un primer acercamiento de la temática en estos negocios, abriendo así el camino para el estudio en otras empresas que por su naturaleza mantienen una forma de gestión lejana al uso estratégico de las tecnologías. 
Entre las futuras líneas de investigación se recomiendan aquellas que exploren tres aspectos: organizacional, operativo y comercial. En el aspecto organizacional, se debe dilucidar cómo las actividades de innovación son más susceptibles cuando se alinean la estructura tecnológica con la cultura, el liderazgo y la resiliencia de las empresas. En la parte operativa, todavía falta explorar sobre los mecanismos tecnológicos que ayuden en la generación y el desarrollo de nuevos productos gastronómicos, servicios y experiencias al comensal. En particular, de micro y pequeñas empresas que están limitadas en recursos financieros, pero que pueden propiciar capacidades a través de la gestión de recursos intangibles como el conocimiento y el talento humano. Por último, la investigación en el aspecto comercial debe examinar las plataformas y los esquemas de difusión de las innovaciones, además de involucrar alianzas y colaboraciones estratégicas con diversos actores, con la finalidad de penetrar de manera más rápida y eficiente en el mercado.

Las limitaciones de investigación fueron el tamaño de la muestra y el corte de la investigación, al tratar un número estadísticamente pequeño de empresas y estudiar transversalmente el fenómeno. Por ello, se sugiere tener cuidado con la generalización de los hallazgos. Otras fueron el uso de un único instrumento para la recolección de los datos y solo abordar la perspectiva de los gerentes. En estudios futuros, se recomienda consultar a los trabajadores y clientes para generar un marco más amplio sobre cómo se desarrolla y percibe la innovación en los restaurantes.

\section{Referencias}

AHN, J. A., y SEO, S. (2018). «Consumer responses to interactive restaurant self-service technology (IRSST): The role of gadget-loving propensity». International Journal of Hospitality Management, 74, pp. 109-121. https://doi.org/10.1016/j.ijhm.2018.02.020

ANNING-DORSON, T. (2017). «Innovation development in service firms: A three-model perspective». International Journal of Services and Operations Management, 28(1), pp. 64-80. https://doi.org/10.1504/ijsom.2017.085905

ARANA-SOLARES, I. A., ORTEGA-JIMÉNEZ, C. H., ALFALLA-LUQUE, R., y PÉREZ-DÍEZ DE LOS RÍOS, J. L. (2018). «Contextual factors intervening in the manufacturing strategy and technology managementperformance relationship». International Journal of Production Economics, 207, pp. 81-95.

https://doi. org/10.1016/j.ijpe.2018.11.003

BADAWY, A. M. (2009). «Technology management simply defined: A tweet plus two characters». Journal of Engineering and Technology Management, 26(4), pp. 219-224.

https://doi.org/10.1016/j. jengtecman.2009.11.001
BARNEY,J.(1991).«Firmresourcesandsustainedcompetitive advantage». Journal of Management, 17(1), pp. 99-120. https://doi.org/10.1177/014920639101700108

BERBEGAL-MIRABENT, J., DE LA TORRE, R., y GIL-DOMÉNECH, D. (2020). «Capitalising new knowledge through R\&D alliances: Evidence from Catalan technology centres». International Journal of Technology Management, 83 (4), pp. 246-268. https://doi.org/10.1504/IJTM.2020.110123

BICEN, P., HUNT, S. D., y MADHAVARAM, S. (2021). "Coopetitive innovation alliance performance: Alliance competence, alliance's market orientation, and relational governance». Journal of Business Research, 123, pp. 23-31. https://doi.org/10.1016/j.jbusres.2020.09.040

BOUNCKEN, R. B., FREDRICH, V., KRAUS, S., y RITALA, P. (2020). «Innovation alliances: Balancing value creation dynamics, competitive intensity and market overlap». Journal of Business Research, 112, pp. 240-247. https://doi.org/10.1016/j.jbusres.2019.10.004

BOUNCKEN, R. B., RATZMANN, M., PESCH, R., y LAUDIEN, S. M. (2018). «Alliances of service firms and manufacturers: Relations and configurations of entrepreneurial orientation and hybrid innovation». Journal of Business Research, 89, pp. 190-197. https://doi.org/10.1016/j.jbusres.2018.01.039

CETINDAMAR, D., PHAAL, R., y PROBERT, D. R. (2009). «Understanding technology management as a dynamic capability: A framework for technology management activities». Technovation, 29(4), pp. 237-246. http://dx.doi.org/10.1016/j.technovation.2008.10.004

CETINDAMAR, D., PHAAL, R., y PROBERT, D. R. (2016a). Technology management activities and tools (2nd ed.). New York: Palgrave Macmillan.

CETINDAMAR, D., PHAAL, R., y PROBERT, D. R. (2016b). «Technology management as a profession and the challenges ahead». Journal of Engineering and Technology Management, 41, pp. 1-13. http://dx.doi.org/10.1016/j.jengtecman.2016.05.001

CHO, M., BONN, M. A., y HAN, S. J. (2019). «Innovation ambidexterity: Balancing exploitation and exploration for startup and established restaurants and impacts upon performance». Industry and Innovation, 27(4), pp. 340362. https://doi.org/10.1080/13662716.2019.1633280

CHOU, S. F., HORNG, J. S., LIU, C. H., HUANG, Y. C., y ZHANG, S. N. (2020). "The critical criteria for innovation entrepreneurship of restaurants: Considering the interrelationship effect of human capital and competitive strategy a case study in Taiwan». Journal of Hospitality and Tourism Management, 42, pp. 222-234. https://doi.org/10.1016/j.jhtm.2020.01.006 
CITUR (2020). Estadísticas nacionales: Económicas PIB. Colombia: Centro de Información Turística de Colombia (CITUR). http://www.citur.gov.co/estadisticas/df pib/ porcentual $/ 45 ? \mathrm{t}=1 \# \mathrm{gsc} . \mathrm{tab}=0$

D'MELLO D. (2020). E-resistance: Making active choices for technology management in an agile working age. En C. Grant y E. Russell (eds.), Agile working and wellbeing in the digital age (pp. 77-90). Suiza: Palgrave Macmillan, Cham.

https://doi.org/10.1007/978-3-030-60283-3 6

DAMANPOUR, F., WALKER, R. M., y AVELLANEDA, C. N. (2009). «Combinative effects of innovation types and organizational performance: A longitudinal study of service organizations». Journal of Management Studies, 46(4), pp. 650-675.

https://doi.org/10.1111/j.1467- 6486.2008.00814.x

DANE (2019). Boletín técnico: Encuesta anual de servicios. Colombia: Departamento Administrativo Nacional de Estadística (DANE). https://www.dane.gov.co/files/ investigaciones/boletines/eas/bp_EAS_2019.pdf

DE MELLO, A. M., MARX, R., y SALERNO, M. (2012). «Organizational structures to support innovation: how do companies decide?» Review of Administration and Innovation, 9(4), pp. 5-21.

https://doi.org/10.5773/rai. v9i4.623

DEDAHANOV, A. T., RHEE, C., y YOON, J. (2017). «Organizational structure and innovation performance». Career Development International, 22(4), pp. 334-350. https://doi.org/10.1108/cdi-12-2016-0234

DELGADO, A., VARGAS, E. E., MONTES, J. M., y RODRÍGUEZ, F. (2016). «Innovation in tourism companies, where are they and where are they going? An approach to the state of knowledge». Intangible Capital, 12(4), pp. 1088-1155. http://dx.doi.org/10.3926/ic.778

DELGADO, A., VARGAS, E. E., RODRÍGUEZ, F., y MONTES, J. M. (2017a). «Gestión tecnológica en restaurantes: Desarrollo y validación de un instrumento de medición». GECONTEC. Revista Internacional de Gestión del Conocimiento y la Tecnología, 5(2), pp. 2340 .

DELGADO, A., VARGAS, E. E., RODRÍGUEZ, F., y MONTES, J. M. (2017b). «Capacidad de innovación en el sector restaurantero: Validación de un instrumento de medición». Multiciencias, 17(1), pp. 26-36.
DELGADO, A., VARGAS, E. E., RODRÍGUEZ, F., y MONTES, J. M. (2018). «Estructura organizacional, capital humano y redes de colaboración: Determinantes de la capacidad de innovación en restaurantes». ADminister, (32), pp. 5-28.

http://dx.doi.org/10.17230/ad-minister.32.1

DIJKSTRA, T. K., y HENSELER, J. (2015). «Consistent partial least squares path modeling». MIS Quarterly, 39(2), pp. 297-316.

https://doi.org/10.25300/MISQ/2015/39.2.02

DIPIETRO, R. (2017). «Restaurant and foodservice research: A critical reflection behind and an optimistic look ahead». International Journal of Contemporary Hospitality Management, 29(4), pp. 1203-1234. https://doi.org/10.1108/IJCHM-01-2016-0046

DREJER, A. (1997). "The discipline of management of technology, based on considerations related to technology». Technovation, 17(5), pp. 253-265. http://dx.doi.org/10.1016/S0166-4972(96)00107-1

FONSECA, T., DE FARIA, P., y LIMA, F. (2019). «Human capital and innovation: The importance of the optimal organizational task structure». Research Policy, 48(3), pp. 616-627. https://doi.org/10.1016/j. respol.2018.10.010

FORNELL, C., y LARCKER, D. F. (1981). «Evaluating structural equation models with unobservable variables and measurement error». Journal of Marketing Research, 18(1), pp. 39-50. https://doi.org/10.2307/3151312

GEISSER, S. (1974). «A predictive approach to the random effects model». Biometrika, 61(1), pp. 101-107. https://doi.org/10.2307/2334290

GRANT, R. M. (1991). «The resource based theory of competitive advantage: Implications for strategy formulation». California Management Review, 33(3), pp. 114-135. https://doi.org/10.1002/smj.4250050207

HAIR, J., HULT, G., RINGLE, C., y SARSTEDT, M. (2017). A primer on partial least square structural equation modeling (PLS-SEM) (2nd ed.). Thousand Oaks, California: Sage.

HAMEED, W. U., NISAR, Q. A., y WU, H. C. (2021). «Relationships between external knowledge, internal innovation, firms' open innovation performance, service innovation and business performance in the Pakistani hotel industry». International Journal of Hospitality Management, 92, p. 102745.

https://doi.org/10.1016/j. ijhm.2020.102745 
HENSELER, J., RINGLE, C. M., y SINKOVICS, R. R. (2009). «The use de partial least squares path modeling in international marketing». Advances in International Marketing, 20, pp. 277-320.

https://doi.org/10.1108/S1474-7979(2009)0000020014

HENSELER, J., RINGLE, C., y SARSTEDT, M. (2016). «Testing measurement invariance of composites using partial least squares». International Marketing Review, 33(3), pp. 405-431.

https://doi.org/10.1108/IMR-09- 2014-0304

HEO, C. Y. (2016). «Exploring group-buying platforms for restaurant revenue management». International Journal of Hospitality Management, 52, pp.154-159. http://dx.doi.org/10.1016/j.ijhm.2015.07.009

HJALAGER, A. M. (1999). «Technology domains and manpower choice in the restaurant sector». New Technology, Work and Employment, 14(1), pp. 62-74. http://dx.doi.org/10.1111/1468-005X.00053

HOHBERGER, J., KRUGER, H., y ALMEIDA, P. (2020). «Does separation hurt? The impact of premature termination of R\&D alliances on knowledge acquisition and innovation». Research Policy, 49(6), p. 103944 https://doi.org/10.1016/j.respol.2020.103944

HOSTELERÍADE ESPAÑA(2019). Anuario de la Hostelería de España, 2019. España: Hostelería de España. https:// hosfrinor.com/wp-content/uploads/2019/12/Anuariode-la-Hosteler\%C3\%ADa-de-España-2019.pdf

HU, L. T., y BENTLER, P. M. (1998). «Fit indices in covariance structure modeling: Sensitivity to underparameterized model misspecification». Psychological Methods, 3(4), pp. 424-453. https://doi.org/10.1037/1082-989X.3.4.424

HUANG, K. E., WU, J. H., LU, S. Y., y LIN, Y. C. (2016). «Innovation and technology creation effects on organizational performance». Journal of Business Research, 69(6), pp. 2187-2192.

http://dx.doi.org/10.1016/j.jbusres.2015.12.028

HUARNG, K. H. (2010). «Essential research in technology management». Journal of Business Research, 63(5), pp. 451-453. https://doi.org/10.1016/j.jbusres.2009.03.017

HURTADO, M. N., JARÍA-CHACÓN, N., y VALLSPASOLA, J. (2017). «Innovation and experimental services: The role of multidisciplinary arts in creative gastronomy toward a research agenda». Dirección y Organización, (61), pp. 32-47.

https://doi.org/10.37610/dyo.v0i61.503

BM (2019). IBM SPSS Statistics for Macintosh (Version 26.0) [software]. Armonk, New York: IBM Corp.
KALKAN, A., BOZKURT, O. C,, y ARMAN, M. (2014). «The impacts of intellectual capital, innovation and organizational strategy on firm performance». ProcediaSocial and Behavioral Sciences, 150, pp. 700-707. https://doi.org/10.1016/j.sbspro.2014.09.025

KANG, H. (2006). «Technology management in services: Knowledge-based vs knowledge-embedded services». Strategic Change, 15, pp. 67-74 http://dx.doi.org/10.1002/jsc.750

KIANTO, A., SÁENZ, J., y ARAMBURU, N. (2017). «Knowledge-based human resource management practices, intellectual capital and innovation». Journal of Business Research, 81, pp. 11-20. https://doi.org/10.1016/j.jbusres.2017.07.018

KIM, J. J., CHOE, J. Y., y HWANG, J. (2020). «Application of consumer innovativeness to the context of robotic restaurants»». International Journal of Contemporary Hospitality Management, 33 (1), pp. 224-242. https://doi.org/10.1108/ijchm-06-2020-0602

KRAWCZYK-DEMBICKA, E. (2017). «Analysis of technology management using the example of the production enterprise from the SME sector». Procedia Engineering, 182, pp. 359-365.

https://doi.org/10.1016/j. proeng.2017.03.112

LEE, C., HALLAK, R., y SARDESHMUKH, S. R. (2016). «Innovation, entrepreneurship, and restaurant performance: a higher-order structural model». Tourism Management, 53, pp. 215-228.

https://doi.org/10.1016/j.tourman.2015.09.017

LEE, C., SARDESHMUKH, S. R., y HALLAK, R. (2016). «A qualitative study of innovation in the restaurant industry». Anatolia, 27(3), pp. 367-376. https://doi.org/10.1080/13032917.2016.1191769

LEUNG, R., y LOO, P. T. (2020). «Co-creating interactive dining experiences via interconnected and interoperable smart technology». Asian Journal of Technology Innovation. https://doi.org/10.1080/19761597.2020.1822748

LIAO, S. (2005). «Technology management methodologies and applications. A literature review from 1995 to 2003». Technovation, 25, pp. 381-393. https://doi.org/10.1016/j.technovation.2003.08.002

MCDERMOTT, C. M., KANG, H., y WALSH, S. (2001). «A framework for technology management in services». IEEE Transactions on Engineering Management, 48(3), pp. 333-341. http://dx.doi.org/10.1109/17.946532 
MCGUIRK, H., LENIHAN, H., y HART, M. (2015). «Measuring the impact of innovative human capital on small firms' propensity to innovate». Research Policy, 44(4), pp. 965-976.

https://doi.org/10.1016/j.respol.2014.11.008

MEDCOF, J. W., y LEE, T. (2017). «The effects of the chief technology officer and firm and industry R\&D intensity on organizational performance». R\&D Management, 47(5), pp. 767-781. https://doi.org/10.1111/radm.12275

MENDOZA, N. V., BARHATE, B., JOHNSTON, A., y JABARKHAIL, S. (2020). "Career trajectories in a technology management-HRD programme». Human Resource Development International. https://doi.org/10.1080/13678868.2020.1852819

MORENO, P., y TEJADA, P. (2019). «Reviewing the progress of information and communication technology in the restaurant industry». Journal of Hospitality and Tourism Technology, 10 (4), pp. 673-688. https://doi.org/10.1108/JHTT-07-2018-0072

NAJIB, M., SEPTIANI, S., y NURLAELA, S. (2020). «The role of innovation, entrepreneurial self-efficacy and local uniqueness on marketing performance in small and medium-sized restaurants». Journal of Foodservice Business Research, 23(6), pp. 499-519. https://doi.org/10.1080/15378020.2020.1803687

NATIONAL RESTAURANT ASSOCIATION (2019). Resturant industry. Estados Unidos: National Restaurant Association https://www.restaurant.org/downloads/pdfs/research/ soi/restaurant_industry_fact_sheet_2019.pdf

NUNNALLY, J., y BERNSTEIN, I. (1994). Psychometric theory (3rd ed.). New York: McGraw-Hill.

PANDA, S., y SHARMA, R. (2020). «Do changes in patent policy influence firms' technology strategy? Evidence from manufacturing in India». Journal of Policy Modeling, en prensa. https://doi.org/10.1016/j.jpolmod.2020.09.002

PARK, S., LEHTO, X., y LEHTO, M. (2021). «Selfservice technology kiosk design for restaurants: An QFD application». International Journal of Hospitality Management, 92, p. 102757.

https://doi.org/10.1016/j.ijhm.2020.102757

PASBAN, M., y NOJEDEH, S. H. (2016). «A review of the role of human capital in the organization». ProcediaSocial and Behavioral Sciences, 230, pp. 249-253. https://doi.org/10.1016/j.sbspro.2016.09.032
PENROSE, E. (1959). The theory of the growth of the firm. Oxford: Basil Blackwell.

http://dx.doi.org/10.1093/0198289774.001.0001

PHAAL, R., FARRUKH, C. J. P., y PROBERT, D. R. (2006). «Technology management tools: Concept, development and application». Technovation, 26(3), pp. 336-344. http://dx.doi.org/10.1016/j.technovation.2005.02.001

PILKINGTON, A., y TEICHERT, T. (2006). «Management of technology: Themes, concepts and relationships». Technovation, 26(3), pp. 288-299. http://dx.doi.org/10.1016/j.technovation.2005.01.009

RAVICHANDRAN, T. (2018). «Exploring the relationships between IT competence, innovation capacity and organizational agility. The Journal of Strategic Information Systems, 27(1), pp. 22-42. https://doi.org/10.1016/j.jsis.2017.07.002

RINGLE, C. M., WENDE, S., y BECKER, J. M. (2015). SmartPLS (version 3.3.3) [software]. Bönningstedt, Germany: SmartPLS GmbH.

RUIZ, M. E., GIL, I., y BERENGUER, G. (2014). «Information and communication technology as a differentiation tool in restaurants». Journal of Foodservice Business Research, 17(5), 410-428. http://dx.doi.org/10.1080/15378020.2014.967639

SALZBERG, A. C., GOUGH, M. Z., y SUEN, I. S. (2019). «Sustainable innovation behavior in restaurants». Journal of Foodservice Business Research, 22(2), pp. 167-190.

https://doi.org/10.1080/15378020.2019.1592655

SCHUMPETER, J. A. (1934). The theory of economic development. Cambridge, MA: Harvard University Press.

SECTUR (2020). Comunicado 155/ 2019. México: Secretaría de Turismo (SECTUR). https://www.gob.mx/ sectur/prensa/el-sector-restaurantero-representa-el-153-por-ciento-del-pib-turistico-en-mexico

SEYITOĞLU, F., e IVANOV, S. (2020). «Understanding the robotic restaurant experience: a multiple case study». Journal of Tourism Futures, en prensa. https://doi.org/10.1108/jtf-04-2020-0070

STONE, M. (1974). «Cross-validatory choice and assessment of statistical predictions». Journal of the Royal Statistical Society, 36(2), pp. 111-147. https://doi.org/10.1111/j.2517-6161.1974.tb00994.x 
SUN, X., LI, H., y GHOSAL, V. (2020). «Firm-level human capital and innovation: Evidence from China». China Economic Review, 59, p. 101388.

https://doi.org/10.1016/j.chieco.2019.101388

SZETO, E. (2000). «Innovation capacity: Working towards a mechanism for improving innovation within an interorganizational network». TQM Magazine, 12(2), pp. 149-158.

TAJVIDI, M., y KARAMI, A. (2015). Innovation capacity. En M. Tajvidi y A. Karami (eds.), Product development strategy (pp. 125-146). Londres: Palgrave Macmillan. https://doi.org/10.1057/9781137501394 5

TEECE, D. J., PETERAF, M., y LEIH, S. (2016). «Dynamic capabilities and organizational agility». California Management Review, 58(4), pp. 13-35. https://doi.org/10.1525/cmr.2016.58.4.13

TEECE, D. J., PISANO, G., y SHUEN, A. (1997). «Dynamic capabilities and strategic management». Strategic Management Journal, 18(7), pp. 509-533.

https://doi.org/10.1002/

(sici)1097-0266(199708)18:7<509::aid-

smj882>3.0.co; $2-\mathrm{z}$

TEECE, D. J., y LEIH, S. (2016). «Uncertainty, innovation, and dynamic capabilities: An introduction». California Management Review, 58(4), pp. 5-12.

https://doi.org/10.1525/cmr.2016.58.4.5

TOU, Y., WATANABE, C., y NEITTAANMÄKI, P. (2019). «Fusion of technology management and financing management - Amazon's transformative endeavor by orchestrating techno-financing systems». Technology in Society, p. 101219.

https://doi.org/10.1016/j.techsoc.2019.101219

TOUR GASTRONÓMICO (2029). Guía de restaurantes. Colombia: Tour Gastronómico. http://www.tourgastronomico.com.co/wp-content/ uploads/2019/06/guiatour2019-2.pdf
TRIPADVISOR (2020). Restaurantes en Medellín. Colombia: Tripadvisor.

VARGAS-SÁNCHEZ, A., y LÓPEZ-GUZMÁN, T. (2018). «Protection of culinary knowledge generation in michelin-starred restaurants. The Spanish case». International Journal of Gastronomy and Food Science, 14 , pp. 27-34.

https://doi.org/10.1016/j.ijgfs.2018.09.001

VILLA, L., y MELO, J. (2015). Panorama actual de la innovación social en Colombia. Washington D. C., Estados Unidos: Banco Interamericano de Desarrollo. https://publications.iadb.org/publications/spanish/ document/Panorama-actual-de-la-innovación-social-enColombia.pdf

WERNERFELT, B. (1984). «A resource-based view of the firm». Strategic Management Journal, 5, pp. 171-180. https://doi.org/10.1177/014920630102700601

WONGLIMPIYARAT, J. (2019). «What is it about strategic implications of using financial models in the process of technology management?» The Journal of High Technology Management Research, 30(1), pp. 82-90. https://doi.org/10.1016/j.hitech.2018.12.001

YUN, J. J., PARK, K., GAUDIO, G. D., y CORTE, V. D. (2020). «Open innovation ecosystems of restaurants: Geographical economics of successful restaurants from three cities». European Planning Studies, 28(12), pp. 2348-2367.

https://doi.org/10.1080/09654313.2020.1721438

ZAHOOR, N., y AL-TABBAA, O. (2020). «Interorganizational collaboration and SMEs' innovation: A systematic review and future research directions». Scandinavian Journal of Management, 36(2), p. 101109. https://doi.org/10.1016/j.scaman.2020.101109 